\title{
Insomnia is Associated with Psychological and Physical Problems in Japanese Male Workers
}

\author{
Machi Suka; Katsumi Yoshida; Hiroki Sugimori; Masashi Nishihara; and Yumi Hatanaka \\ Department of Preventive Medicine, St. Marianna University School of Medicine
}

\section{ABSTRACT}

Objectives To elucidate sociodemographic, lifestyle, psychological, and physical factors associated with two subtypes of insomnia in Japanese male workers.

Design A cross-sectional study using the annual health examination database of a Japanese company.

\section{Setting N/A}

Participants Middle-aged male participants in the 1998 health examination at an employee health management center of a Japanese company $(n=22,185)$.

Main outcome measures Self-reported insomnia, including difficulty initiating sleep (DIS) and difficulty maintaining sleep (DMS).

Results In the young age ( $<45$ years old) group: Significant higher odds ratio (OR) for DIS was found in living alone, shift work, smoking, lack of having three meals, psychological problems, and hypertension, with significant lower OR in overtime work and alcohol drinking. Significant higher OR for DMS was found in living alone, alcohol drinking, psychological problems, and hypertension, with significant lower OR in overtime work. In the old age $(\geq 45$ years old) group: Significant higher OR for DIS was found in living alone, smoking, lack of having three meals, lack of exercise, and psychological problems, with significant lower OR in overtime work, alcohol drinking, and hypertension. Significant higher OR for DMS was found in alcohol drinking, lack of walk, psychological problems, and diabetes, with significant lower OR in overtime work and smoking.

Conclusions Insomnia was associated with psychological and physical problems, besides lifestyles, in Japanese male workers.

Key Words Cross-sectional Study; Insomnia; Hypertension; Lifestyle; Psychological Complaint

\section{INTRODUCTION}

Insomnia is one of the most common complaints in worksite, as well as in the general population. It has a great influence on daily life functions and can cause accidents. ${ }^{[1]}$ In addition, recent prospective studies suggested that short or long sleep duration or insomnia might increase the risks of cardiovascular disease ${ }^{[2],[3]}$ and mortality. ${ }^{[4], ~[5]}$

The prevalence of insomnia has been found to be associated with a number of sociodemographic, lifestyle, psychological, and physical factors. Sociodemographic factors include old age, ${ }^{[6]-[10]}$ female sex, ${ }^{[6], ~[9]-[1]]}$ single, ${ }^{[8],[11]}$ low education, ${ }^{[10], ~[11]}$ low income, ${ }^{[10], ~[11] ~ i n o c c u p a t i o n, ~}{ }^{[7], ~[8], ~[11] ~}$ and shift work. ${ }^{[12], ~[13] ~}$ Lifestyle factors include smoking, ${ }^{[1]]}$ alcohol drinking, ${ }^{[10], ~[12], ~[14] ~}$ and physical inactivity. ${ }^{[6], ~[7], ~[12], ~[14], ~[15] ~ P s y c h o l o g i c a l ~ f a c t o r s ~}$ include anxiety, ${ }^{[10], ~[13]}$ depression, ${ }^{[10], ~[16]}$ and stress. ${ }^{[7], ~[11], ~[13], ~[15], ~[17], ~[18] ~}$ Physical factors include overweight, ${ }^{[14]}$ pain, ${ }^{[11]}$ disability, ${ }^{[1]}$ in addition to specific medical conditions (e.g. musculoskeletal disease, digestive disease, circulatory disease, respiratory disease, diabetes, migraine). ${ }^{[6],[11],[14],[16],[19]}$ These factors might have

Received: May 24, 2003

Address; 2-16-1, Sugao, Miyamae-ku, Kawasaki, Kanagawa, 2168511 Japan. TEL: 81-44-977-8111 (ex.3428), FAX: 81-44-977-8356, E-mail: strong associations or interactions with one another. However, only a few studies examined such potential associated factors together in multivariate analyses, ${ }^{[7],[10],[1]]}$ which are commonly used for adjusting for confounding factors. Moreover, the factors associated with insomnia were not always identical among studies. They are likely to be affected by differences in social and cultural backgrounds between western countries and Japan.

Using the annual health examination database of a Japanese company, a large-scale cross-sectional study was conducted to examine the multivariate associations with insomnia. Our findings may afford a new prospect on sociodemographic, lifestyle, psychological, and physical factors associated with insomnia in Japanese male workers.

\section{SUBJECTS AND METHODS}

Annual health examination database was accumulated from an employee health management center of a Japanese telecommunication company. ${ }^{[20]}$ The participants in the annual health examinations were asked to complete a structured questionnaire about their medical history, physiological and psychological complaints, and daily life. Systolic and diastolic blood pressures were measured after five minutes of rest in a sitting position by well-trained nurses. Blood samples (fasting) were taken for measurements of biochemical and hematological variables. The above processes were conducted according to the guidelines established by the employee health management center. All the available data were stored in the database.

Subjects of this study were 22,185 eligible male office workers and participants in the 1998 health examination, (1) who were aged 30 to 59 years, (2) who gave answers to three questions about insomnia in the questionnaire, and (3) who had measurements of blood pressure, total cholesterol, triglyceride, and fasting blood glucose available.

The three questions about insomnia were on "falling asleep with difficulty", "awakening many times in the night", and "awakening once in the night and falling asleep again with difficulty". Each question had three possible options of 'always', 'sometimes', and 'never'. In this study, insomnia was classified into two subtypes, that is, "difficulty initiating sleep; DIS" and "difficulty maintaining sleep; DMS". The presence of DIS was defined by an affirmative answer ('always' or 'sometimes') to the "falling asleep with difficulty". That of DMS was defined by an affirmative answer ('always' or 'sometimes') to the "awakening many times in the night" and The "awakening once in the night and falling asleep again with difficulty".

As for lifestyles, partly sociodemographics, the following eight variables were included: "household" ('live alone' vs. 'live with family'), "work schedule” ('shift work' vs. 'day work'), "overtime work" (hours per month; $\geq 30$ vs. $<30$ ), "smoking" (cigarettes per 
day; $\geq 20$ and $<20$ vs. never), "alcohol drinking" (days per week; 6-7 and 2-5 vs. 0-1), "having three meals" ('never' and 'some days' vs. 'every day'), "exercise” ('irregularly or never' vs. 'regularly'), and "walk" (hours per day; $<1$ vs. $\geq 1$ ). As for psychological problems, the following four variables were included: "stress of work" ('feel' vs. 'not feel'), "worries of relationships" ('feel' vs. 'not feel'), "depression" ('feel' vs. 'not feel')), and "loss of patience" ('feel' vs. 'not feel'). As for physical problems, the following three variables were included: "hypertension; HT" (systolic blood pressure $\geq 140 \mathrm{mmHg}$ and/or diastolic blood pressure $\geq 90 \mathrm{mmHg}$ or taking medication), "hyperlipidemia; HL" (total cholesterol $\geq 5.7 \mathrm{mmol} / l ; 220 \mathrm{mg} / \mathrm{d} l$ and/or triglyceride $\geq 1.7$ $\mathrm{mmol} / l ; 150 \mathrm{mg} / \mathrm{d} l$ ), and "diabetes; DM" (fasting blood glucose $\geq 7.0 \mathrm{mmol} / l ; 126 \mathrm{mg} / \mathrm{d} l$ ). These physical factors were selected because of main components of metabolic syndrome. ${ }^{[21]}$ Body mass was regarded as a confounding factor, and its effect was adjusted in the multivariate analyses.

We did not obtain informed consent from each participant. However, we paid attention to protection of anonymity and confidentiality of the available data.

\section{STATISTICAL ANALYSES}

The subjects were divided into two age groups of $<45$ and $\geq 45$ years old. The associations between respective variables and two subtypes of insomnia were examined using chi-square tests. Adjusted odds ratios (ORs) and their corresponding 95\% confidence intervals (CIs) for DIS and DMS were calculated using multiple logistic regression models, which simultaneously included (1) body mass, sleep hours, lifestyles, and psychological problems or (2) body mass, sleep hours, lifestyles, psychological problems, and physical problems. In these models, body mass were categorized as $<18.6,18.6-24.9,25.0-29.9$, and $30.0 \leq \mathrm{kg} / \mathrm{m}^{2}$, and sleep hours were categorized as $<5,5,6,7$, and $8 \leq$ hours per night. All of the statistical analyses were performed using version 8.02 software from Statistical Analysis Systems (Cary, NC).

\section{RESULTS}

Table1 shows the distributions of DIS and DMS. The prevalence of DMS was significantly higher in the young group (15\%) than in the old age group (19\%), while in the prevalence of DIS, there was no significant difference between the age groups $(12 \%$ vs. $12 \%)$.

Table 2 shows the associations between lifestyles and two subtypes of insomnia. In both the young and old age groups, almost all of the lifestyles were associated with higher prevalence of DIS and/or DMS. By way of exception, in the old age group, "alcohol drinking" was associated with lower prevalence of DIS, and "smoking" was associated with lower prevalence of DMS.

Table 3 shows the associations between psychological problems and two subtypes of insomnia. In both the young and old age groups, all of the psychological problems were significantly associated with higher prevalence of DIS and DMS.

Table 4 shows the associations between physical problems and two subtypes of insomnia. In the young age group, both HT and HL were associated with higher prevalence of DIS and DMS. In the old age group, HT was associated with both lower prevalence of DIS and higher prevalence of DMS. DM was associated with
Table 1 Distributions of DIS and DMS.

\begin{tabular}{lccccccc}
\hline \multirow{2}{*}{$\begin{array}{l}\text { Age } \\
\text { (y.o.) }\end{array}$} & \multicolumn{3}{c}{ DIS } & & \multicolumn{3}{c}{ DMS } \\
\cline { 2 - 4 } \cline { 6 - 8 } & never & sometimes & always & & never & sometimes & always \\
\hline$<45$ & 7,214 & 839 & 189 & & 6,991 & 1,050 & 201 \\
& $88 \%$ & $10 \%$ & $2 \%$ & & $85 \%$ & $13 \%$ & $2 \%$ \\
$\geq 45$ & 12,219 & 1,308 & 416 & & 11,285 & 2,076 & 582 \\
& $88 \%$ & $9 \%$ & $3 \%$ & & $81 \%$ & $15 \%$ & $4 \%$ \\
\hline
\end{tabular}

DIS=difficulty initiating sleep, DMS = difficulty maintaining sleep

\section{higher prevalence of DMS.}

Table 5 shows the results of multiple logistic regression analyses. In the young age group, as for DIS, significantly higher OR was found in "household", "work schedule", "smoking", "having three meals", and all of the psychological problems, while significantly lower OR was found in "overtime work" and "alcohol drinking". As for DMS, significantly higher OR was found in "household", "alcohol drinking", and all of the psychological problems, while significantly lower OR was found in "overtime work". The inclusion of physical problems into the model had few influences on the associations of lifestyles and psychological problems (Model 2). After adjusting for body mass, sleep hours, lifestyles, and psychological problems, HT was significantly associated with both DIS and DMS. In the old age group, as for DIS, significantly higher OR was found in "household", "smoking", "having three meals", "exercise", and all of the psychological problems, while significantly lower OR was found in "overtime work" and "alcohol drinking". As for DMS, significantly higher OR was found in "alcohol drinking", "walk", and all of the psychological problems, while significantly lower OR was found in "overtime work" and "smoking". The inclusion of physical problems into the model had few influences on the associations of lifestyles and psychological problems (Model 2). After adjusting for body mass, sleep hours, lifestyles, and psychological problems, DM was significantly associated with DMS. HT was significantly but inversely associated with DIS.

\section{DISCUSSION}

Using the annual health examination database of a Japanese company, a large-scale cross-sectional study was conducted to examine the multivariate associations with insomnia. As the results of multiple logistic regression analyses, we elucidated sociodemographic, lifestyle, psychological, and physical factors associated with two subtypes of insomnia in Japanese male workers. The effect of each factor on the prevalence of DIS or DMS was different between the young $(<45$ years old) and old $(\geq 45$ years old) age groups. To our knowledge, this is the first report on such a wide range of factors associated with insomnia in worksite.

\section{Lifestyles and insomnia}

As for smoking and alcohol drinking, we found interesting patterns in the associations with two subtypes of insomnia. Smoking had an unfavorable effect on the prevalence of DIS in contrast to a favorable effect on DMS. On the other hand, alcohol drinking had an unfavorable effect on the prevalence of DMS in contrast to a favorable effect on DIS. Several studies showed that smoking or alcohol drinking was associated with higher prevalence of insomnia, ${ }^{[10\}-[12],[14]}$ but they did not deal with subtypes of insomnia. Kim et $a l^{[7]}$ reported no significant effect of smoking or alcohol drink- 


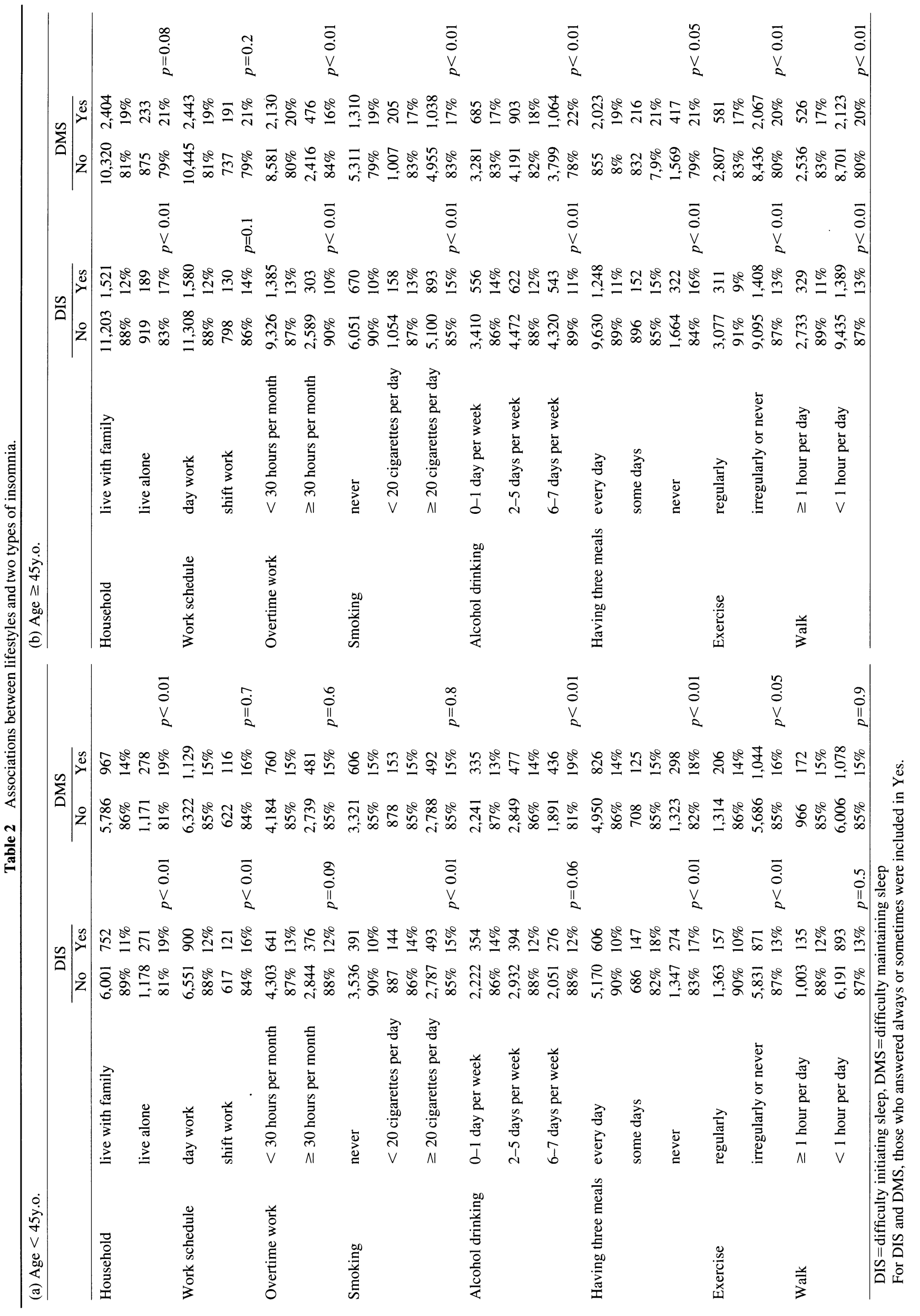




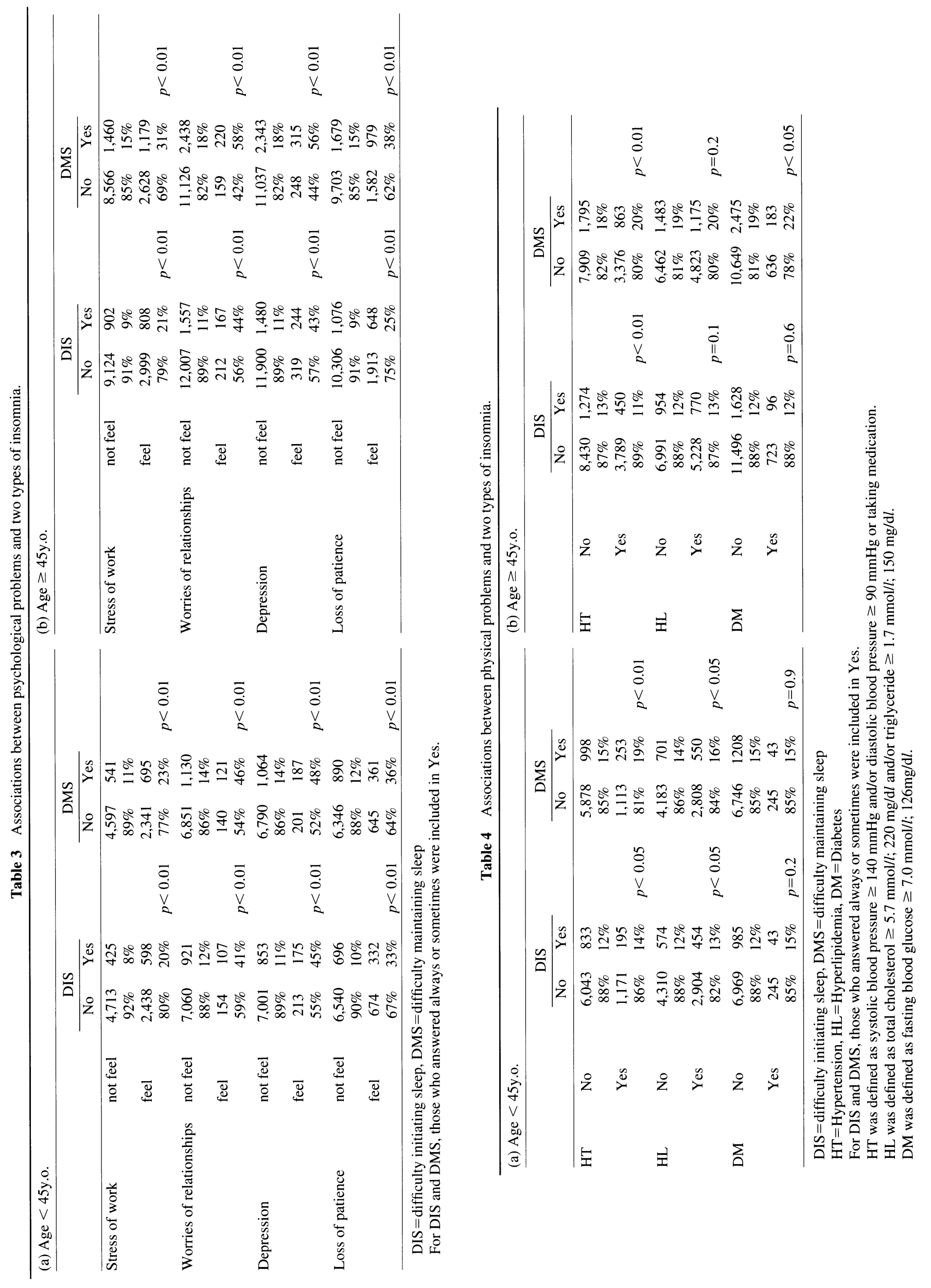




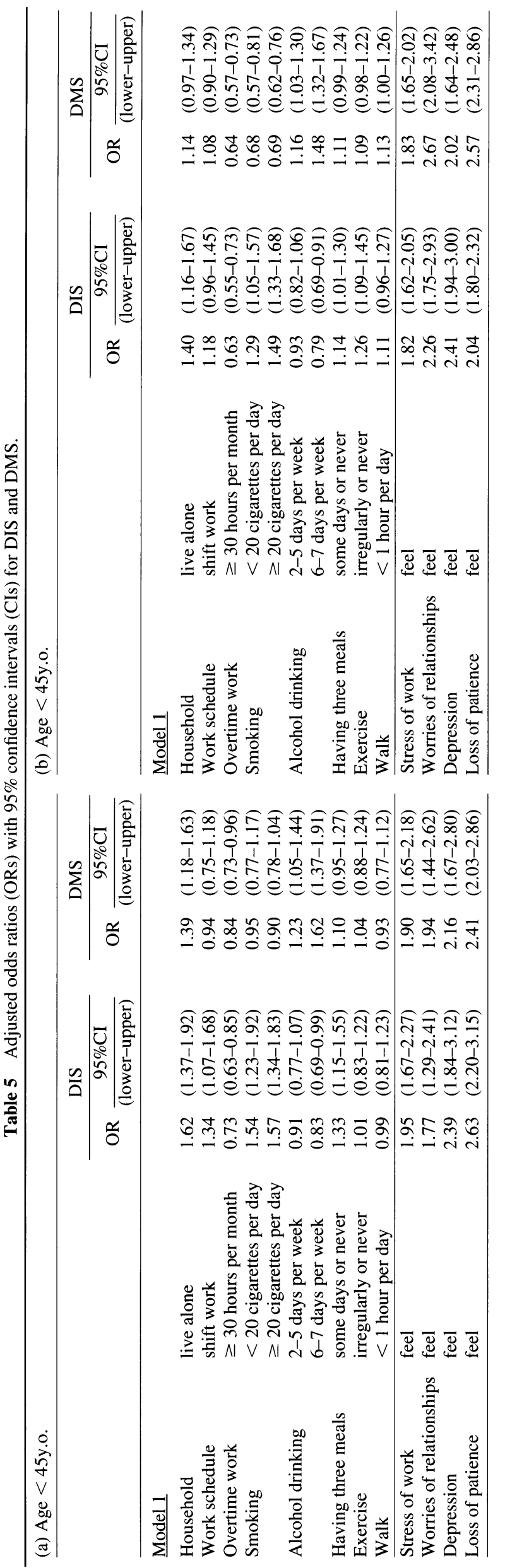

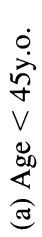

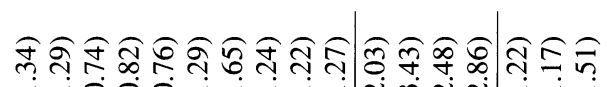

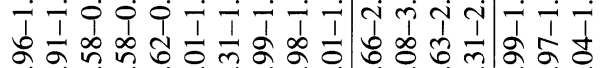

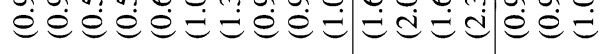

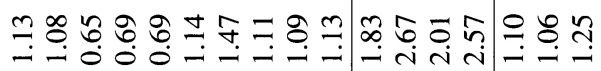

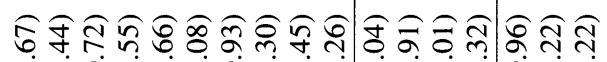

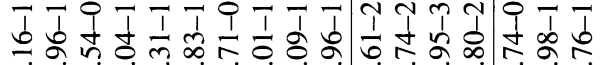

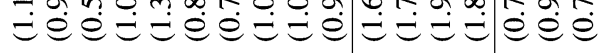

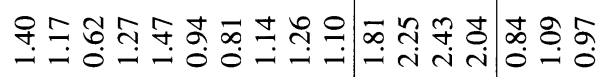

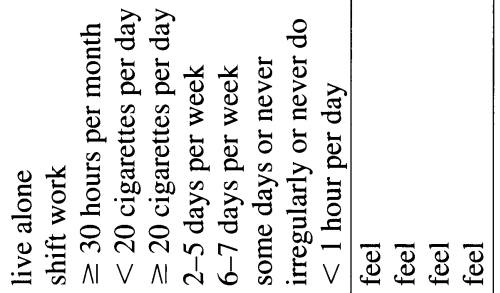

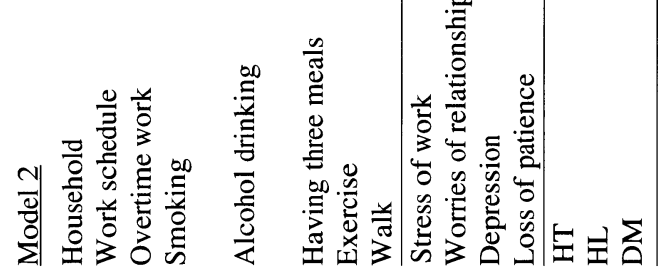

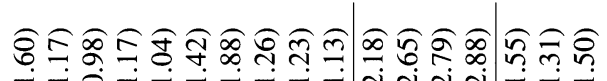

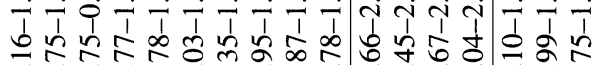

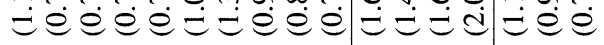

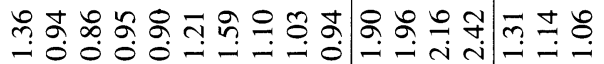

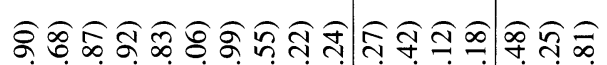

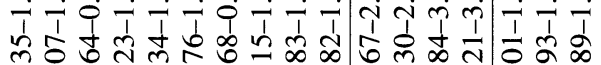

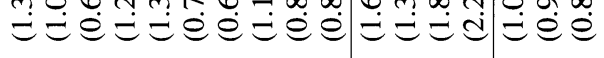

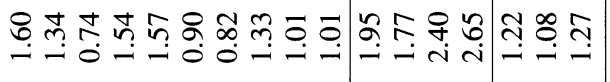

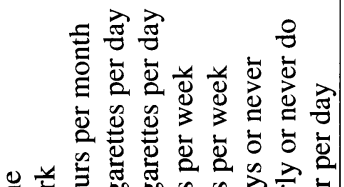

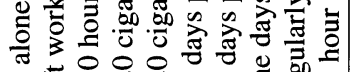

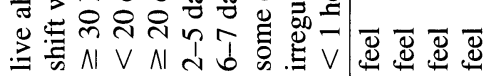

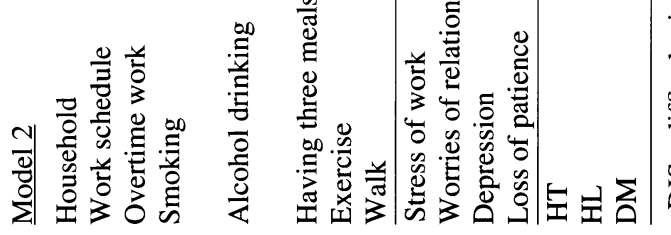

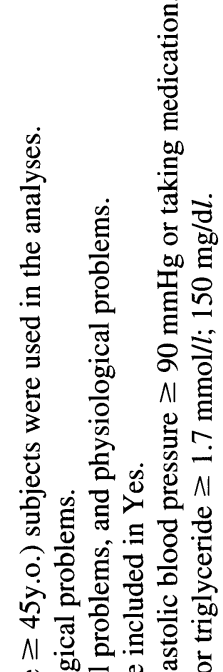
造

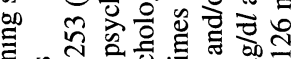

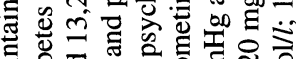

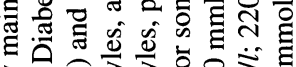

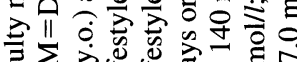

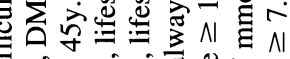

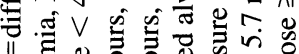
II $\sum: \leq 20$

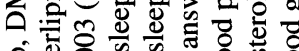

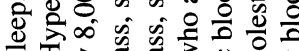

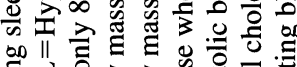

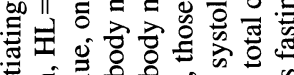

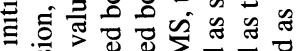

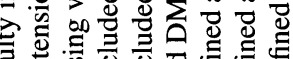

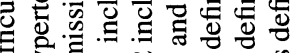
空

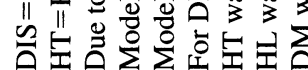


ing on any subtypes of insomnia in the Japanese general population. Because of the inclusion of both men and women, their findings are likely to be affected by obvious sex differences in smoking and drinking habits. ${ }^{[10]}$ Nicotine, as well as caffeine, is known to be a stimulant. ${ }^{[1]}$ The unfavorable effect of smoking on DIS seems to be reasonable. Alcohol is often used for facilitating sleep onset, but this use can cause awakening later in the night ${ }^{[1]}$. The contrasting effects of alcohol drinking on two subtypes of insomnia seem to be reasonable. However, it is difficult to explain about the favorable effect of smoking on DMS. As for the overall prevalence of insomnia, Harma et al. ${ }^{[12]}$ and Fabisitz et al. ${ }^{[22]}$ reported lower OR in smoking with higher OR in alcohol drinking. The effects of smoking and alcohol drinking on two subtypes of insomnia should be again examined in further study.

In the old age group, lack of exercise was associated with higher prevalence of DIS, and lack of walk was associated with higher prevalence of DMS. These results support the unfavorable effect of physical inactivity on the prevalence of insomnia in other studies. ${ }^{[6], ~[7], ~[12], ~[14], ~[15] ~ S e v e r a l ~ e x p e r i m e n t a l ~ s t u d i e s ~ h a v e ~ r e v e a l e d ~ t h a t ~}$ exercise in the daytime promotes better sleep. ${ }^{[23]}$ Although no significant effect of exercise or walk on any subtypes of insomnia was found in the young age group, it is worth pointing out that increase in physical activity may prevent insomnia to some extent.

Having three meals was associated with lower prevalence of DIS in both the young and old age groups. Ohida et al. ${ }^{[24]}$ reported that irregular eating habits (i.e. lack of having three meals) were associated with both subjective sleep insufficiency and short sleep duration. However, little is known about the associations of having three meals and the subtypes of insomnia. It is likely that those who had three meals had healthy lifestyles, besides having three meals, and kept good hours. The effect of having three meals on the prevalence of DIS should be again examined in further study, taking into account a wider range of lifestyle factors.

Living alone was associated with higher prevalence of DIS and DMS in both the young and old age groups. Those who live alone were either single or temporal dispatch without family. Several studies showed that single was associated with higher prevalence of insomnia. ${ }^{[8],[11]}$ The unfavorable effects of living alone on the subtypes of insomnia might suggest the effects of temporal dispatch without family, besides those of single.

Shift workers often complain about insomnia. Several studies showed the combined effects of shift work and lifestyle factors on the prevalence of insomnia. ${ }^{[12],[13]}$ Although shift work was associated with only higher prevalence of DIS in the young age group, the healthy shift worker effect ${ }^{[25]}$ might enable health consequence of shift work including insomnia to be underestimated. Contrary to our expectation, overtime work was associated with lower prevalence of DIS and DMS in both the young and old age groups. Because of self-reported data on overtime work, our findings are likely to be affected by unreported overtime work. The effects of work-related factors on the subtypes of insomnia should be again examined in further study.

\section{- Psychological problems and insomnia}

All of the psychological problems (i.e. stress of work, worries of relationships, depression, and loss of patience) were significantly associated with higher prevalence of DIS and DMS in both the young and old age groups; the three highest OR were found in the psychological problems. These results support the significant associations between psychological factors and insomnia in other studies. ${ }^{[7], ~[10], ~[11], ~[13], ~[15]-[18] ~ T h e ~ e x a m i n e d ~ p s y c h o l o g i c a l ~ f a c t o r s ~}$ varied among studies. Moreover, the unfavorable effects of psychological factors on the prevalence of insomnia might possibly be affected by the ability to cope with stress ${ }^{[7]}$ and social support from family, friends, and coworkers. ${ }^{[18]}$ Further studies, taking into account a wider range of psychological factors, may provide useful information for preventive measures against insomnia in worksite.

\section{- Physical problems and insomnia}

HT was associated with higher prevalence of DIS and DMS in the young age group, and contrary to our expectation, this physical factor was also associated with lower prevalence of DIS in the old age group. Some antihypertensive drugs can cause insomnia. ${ }^{[26]}$ Due to the inclusion of patients who were taking medication, our findings are likely to be affected by drug-induced insomnia. ${ }^{[19]}$ However, the inverse association between HT and DIS in the old age group was found even when HT was determined only by measurement of blood pressure (data not shown).

HT has been found to be associated with other kinds of sleep disturbances, that is, sleep-disordered breathing and sleep apnea. ${ }^{[27]-[30]}$ However, only a few studies examined the association between HT and insomnia. Janson et al. ${ }^{[14]}$ reported no significant effect of HT on the prevalence of insomnia, but they did not deal with subtypes of insomnia. Gislason et al. ${ }^{[19]}$ reported that HT was associated with higher prevalence of DIS and DMS, but they did not adjust for confounding factors. It is difficult to get a conclusive answer about the associations between HT and the subtypes of insomnia based on these findings and our finding.

DM was significantly associated with higher prevalence of DMS in the old age group. As is the case with HT, only a few studies examined the association between DM and insomnia. Janson et al ${ }^{[14]}$ reported no significant effect of DM on the prevalence of insomnia, but they did not deal with subtypes of insomnia. Gislason et $a l .{ }^{[19]}$ reported that DM was associated with higher prevalence of DIS and DMS, but they did not adjust for confounding factors. It is difficult to get a conclusive answer about the associations between DM and the subtypes of insomnia based on these findings and our finding. The case-control study by Shrighar et $a l .{ }^{[31]}$ showed that sleep disturbances in diabetic patients were associated with physical discomfort from cough, dyspnea, nocturnal cramps, paresthesia, and burning of soles. Further studies, taking into account diabetic symptoms, may reveal the details of association between DM and insomnia.

Both HT and DM are known to be main components of metabolic syndrome. ${ }^{[21]}$ Recent prospective studies showed that short or long sleep duration or insomnia might increase the risk of cardiovascular disease. ${ }^{[2],[3]}$ Although the three- or four-way relationships are still unclear, it is worth pointing out that the potential interaction between HT or DM and insomnia may lead to excessive cardiovascular morbidity and mortality.

Thanks to the use of annual health examination database, we obtained data on a wide range of factors, including sociodemographic, lifestyle, psychological, and physical factors. Moreover, the physical problems (i.e. HT, HL, and DM) were determined by actual measurement rather than by self-reported data. For these facts, this study may be prior to other studies. However, this study has the following potential limitations. First, acute/transient insomnia was not eliminated from insomnia. Acute insomnia (lasting a few weeks) is often caused by a transient stress, while 
chronic insomnia (at least three nights per week; lasting a month or more) may be caused by multiple factors and often occurs in conjunction with other health problem. ${ }^{[1]}$ The inclusion of acute insomnia into the case might enable the unfavorable effects of psychological problems on the prevalence of insomnia to be overestimated. However, the answers to the questions about insomnia were expressed in the frequency of complaints, and it is more likely that those who answered 'always' or 'sometimes' had chronic insomnia rather than acute insomnia. Second, the effects of sleep environment were not taken into account. Several studies showed uncomfortable bedroom and noisy residential area were associated with insomnia. ${ }^{[10],{ }^{[32]}}$ Finally, the cross-sectional design makes difficult to determine the causal associations between psychological or physical problems and insomnia. Further studies may be required to confirm our findings in other populations and to address the question of whether insomnia causes psychological and physical problems or vice versa in a follow-up design.

\section{CONCLUSION}

Using the annual health examination database of a Japanese company, a large-scale cross-sectional study was conducted to examine the multivariate associations with insomnia. Insomnia was associated with psychological and physical problems, besides lifestyles, in Japanese male workers.

\section{REFERENCES}

[1] National Heart, Lung, Blood Institute Working Group on Insomnia: Insomnia: Assessment and Management in Primary Care, Am Fam Physician, 59: 3029-3038, 1999

[2] Mallon, L., Broman, J.E., Hetta, J.: Sleep Complaints Predict Coronary Artery Disease Mortality in Males: A 12-year Follow-up Study of a Middle-aged Swedish Population, J Intern Med, 251: 207-216, 2002

[3] Ayas, N.T., White, D.P., Manson, J.E., Stampfer, M.J., Speizer, F.E., Malhotra, A., Hu, F.B.: A Prospective Study of Sleep Duration and Coronary Heart Disease in Women, Arch Intern Med, 163: 205-209, 2003

[4] Kojima, M., Wakai, K., Kawamura, T., Tamakoshi, A., Aoki, R., Lin, Y., Nakayama, T., Horibe, H., Aoki, N., Ohno, Y.: Sleep Pattern and Total Mortality: A 12-Year Follow-up Study in Japan, J Epidemiol, 10: 87-93, 2000

[5] Kripke, D.F., Garfinkel, L., Wingard, D.L., Klauber, M.R., Marler, M.R.: Mortality Associated with Sleep Duration and Insomnia, Arch Gen Psychiatry, 59: 131-136, 2002

[6] Klink, M.E., Quan, S.F., Kaltenborn, W.T., Lebowitz, M.D.: Risk Factors Associated with Complaints of Insomnia in a General Adult Population, Arch Intern Med, 152: 1634-1637, 1992

[7] Kim, K., Uchiyama, M., Okawa, M., Liu, X., Ogihara, R.: An Epidemiological Study of Insomnia Among the Japanese General Population, Sleep, 23: 41-47, 2000

[8] Doi, Y., Minowa, M., Okawa, M., Uchiyama, M.: Prevalence of Sleep Disturbance and Hypnotic Medication Use in Relation to Sociodemographic Factors in the General Japanese Adult Population, J Epidemiol, 10: 79-86, 2000

[9] Doi, Y., Minowa, M., Uchiyama, M., Okawa, M.: Subjective Sleep Quality and Sleep Problems in the General Japanese Adult Population, Psychi Clin Neurosci, 55: 213-215, 2001

[10] Li, R.H., Wing, Y.K., Ho, S.C., Fong, S.Y.: Gender Differences in Insomnia: A Study in the Hong Kong Chinese Population, J Psychosom Res, 53: 601-609, 2002

[11] Sutton, D.A., Moldofsky, H., Badley, E.M.: Insomnia and Health Problems in Canadians, Sleep, 24: 665-670, 2001
[12] Harma, M., Tenkanen, L., Sjoblom, T., Alikoski, T., Heinsalmi, P.: Combined Effects of Shift Work and Life-style on the Prevalence of Insomnia, Sleep Deprivation and Daytime Sleepiness, Scand J Work Environ Health, 24: 300-307, 1998

[13] Ohida, T., Takemura, S., Nozaki, N., Kawahara, K., Sugie, T., Uehata, T.: The Influence of Lifestyle and Night-shift Work on Sleep Problems Among Female Hospital Nurses in Japan, Nippon Kosyu Eisei Zasshi, 48: 595-603, 2000 (in Japanese)

[14] Janson, C., Lindberg, E., Gislason, T., Elmasry, A., Boman, G.: Insomnia in Men: A 10-Year Prospective Population Based Study, Sleep, 24: 425-430, 2001

[15] Kim, K., Uchiyama, M., Okawa, M., Doi, Y., Oida, T., Minowa, M., Ogihara, R.: Lifestyles and Sleep Disorders Among the Japanese General Population, Psychi Clin Neurosci, 53: 269-270, 1999

[16] Kim, K., Uchiyama, M., Liu, X., Shibui, K., Ohida, T., Ogihara, R., Okawa, M.: Somatic and Psychological Complaints and Their Correlates with Insomnia in the Japanese General Population, Psychosom Med, 63: 441-446, 2001

[17] Tachibana, H., Izumi, T., Honda, S., Takemoto, T.: The Prevalence and Pattern of Insomnia in Japanese Industrial Workers: Relationship between Psychosocial Stress and Type of Insomnia, Psychi Clin Neurosci, 52: 397-402, 1998

[18] Nakata, A., Haratani, T., Kawakami, N., Takahashi, M., Shimizu, H., Miki, A., Kobayashi, F., Araki, S.: Relationship between Perceived Job Stress and Sleep Habits in Daytime Female Workers, Kodo Igaku Kenkyu, 7: 39-46, 2001 (in Japanese)

[19] Gislason, T., Almqvist, M.: Somatic Diseases and Sleep Complaints, Act Med Scand, 221: 475-481, 1987

[20] Suka, M., Sugimori, H., Yoshida, K.: Application of the Updated Framingham Risk Score to Japanese Men, Hypertension Research, 24: 685-689, 2001

[21] Suka, M., Sugimori, H., Yoshida, K.: MRFS: Multiple Risk Factor Syndrome, Health Sciences, 16: 188-200, 2000 (in Japanese)

[22] Fabsitz, R.R., Sholinsky, P., Goldberg, J.: Correlates of Sleep Problems Among Men: The Vietnam Era Twin Registry, J Sleep Res, 6: 50-56, 1997

[23] O'Connor, P.J., Youngstedt, S.D.: Influence of Exercise on Human Sleep, Exerc Sport Sci Rev, 23: 105-134, 1995

[24] Ohida, T., Kamal, A.M., Uchiyama, M., Kim, K., Takemura, S., Sone, T., Ishii, T.: The Influence of Lifestyle and Health Status Factors on Sleep Loss Among the Japanese General Population, Sleep, 24: 333-338, 2001

[25] Knutsson, A., Akerstedt, T.: The Healthy-worker Effect: Selfselection Among Swedish Shift Workers, Work Stress, 6: 163-167, 1992

[26] Kupper, D.J., Reynolds, C.F.: Management of Insomnia, New Eng J Med, 336: 341-345, 1997

[27] Nieto, F.J., Young, T.B., Lind, B.K., Shahar, E., Samet, J.M., Redline, S., D’Agostino, R.B., Newman, A.B., Lebowitz, M.D., Pickering, T.G.: Association of Sleep-disordered Breathing, Sleep Apnea, and Hypertension in a Large Community-based Study, JAMA, 283: 1829-1836, 2000

[28] Ohayon, M.M., Guilleminault, C., Priest, R.G., Zulley, J., Smirne, S.: Is Sleep-disordered Breathing an Independent Risk Factor for Hypertension in the General Population (13,057 subjects)?, J Psychosom Res, 48: 593-601, 2000

[29] Bixler, E.O., Vgontzas, A.N., Lin, H.M., Have, T.T., Leiby, B.E., Vela-Bueno, A., Kales, A.: Association of Hypertension and Sleep-disturbed Breathing, Arch Intern Med, 160: 2289-2295, 2000

[30] Sjostrom, C., Lindberg, E., Elmasry, A., Hagg, A., Svardsudd, K., Janson, C.: Prevalence of Sleep Apnoea and Snoring in Hypertensive Men: A Population Based Study, Thorax, 57: 602-607, 2002

[31] Sridhar, G.R., Medhu, K.: Prevalence of Sleep Disturbances in Diabetes Mellitus, Diabetes Res Clin Pract, 23: 183-186, 1994

[32] Ishigooka, J., Suzuki, M., Isawa, S., Muraoka, H., Murasaki, M., Okawa, M.: Epidemiological Study on Sleep Habits and Insomnia of New Outpatients Visiting General Hospitals in Japan, Psychi Clin Neurosci, 53: 515-522, 1999 